Brit. J. prev. soc. Med. (1965), 19, 8-17

\title{
OBSERVATIONS ON TWINNING IN NEW YORK STATE
}

\author{
BY \\ ALAN M. GITTELSOHN AND SAMUEL MILHAM, JR. \\ From the Department of Biostatistics, Johns Hopkins School of Hygiene and Public Health, Baltimore, Maryland \\ and the New York State Department of Health, Albany, New York
}

The present investigation of the incidence of human twinning related to gestational and demographic characteristics is one of a series of birth-rate studies of all births recorded in New York-State, excluding New York City. The twin births were selected from a population of approximately two million maternities registered during the 11-year period. A previous communication (Gittelsohn and Milham, 1964) described the information available for each pair of twins, and the linkage methodology for updating maternity records from the individual birth records of members of pairs of twins.

The phenomenon of twinning in man has long aroused the curiosity and speculation accorded to the rare event. Recognition of the two distinct embryological processes whereby twins arise has stimulated research workers to explore various aspects of the condition. In effect, monozygotic and dizygotic twinnings are naturally occurring mechanisms which simulate controlled experimentation. Monozygotic twins have been assumed to be genetically identical, while dizygotic twins have been regarded as being no more alike, genetically, than siblings. By measuring differentials in the concordance of qualitative traits, and by correlating quantitative traits within the two types of twins, inferences have been drawn about the relative roles of heredity and environment in the development and expression of given phenotypes.

From an alternative viewpoint, it has long been recognized that twins are at greater risk of perinatal death than single births. The various aspects of the lowered viability of twins during the pre-natal and post-natal period have been described in a number of commentaries based on vital statistics and hospital records (Barr and Stevenson, 1961 ; Lowe and Record, 1951 ; Record, Gibson, and McKeown, 1952; Karn, 1953; Yerushalmy and Sheerar, 1940). The excess mortality risk of twins over single births has been ascribed to increased occurrence of such complications as prematurity, toxaemia, placenta praevia, polyhydramnios, premature separation of the placenta, and malpresentation. In terms of the uniparous condition characteristic of human birth, binovular and uniovular twinning may be regarded as reproductive anomalies leading to lowered fitness. Several teratogenic agents and varied physical conditions have been observed to result in a higher incidence of monozygotic twinning along with various types of developmental abnormalities in experimental animals (Witschi, 1952). Alternatively, the high loss rates of twins may be indicative of maladaptation of the human uterus to the simultaneous gestation of more than one foetus. Suggestions of underlyingo causal mechanisms may be obtained through study? of differential loss rates by plurality and by zygosity groups.

\section{SOURCES OF DATA}

Present observations include all twin births registered and/or resident in New York State, excluding New York City, during the period 1950 to 1960 . State law requires the reporting of all live births and all products of conception with gestation lengths of 20 or more weeks. Matching individual twin birth records were combined into a single twinship record and subsequently updated with mortality information through the first year of life. Twin pairs with a single birth certificate were excluded from consideration. The under-enumeration error thereby committed is small, since such an occurrence is quite rare, such cases usually representing parasitic twinning (i.e. cases of unequal or asymmetrical conjoined twins). Of much greater significance is the underregistration of stillborn foetuses around the period of 20 weeks' gestation. Within the present descriptive context, the major advantage of the birth and death reporting system as an information source lies in its inclusiveness from a population standpoint. In New York State less than 1 per cent. of all births occur outside hospitals. Excluding early foetal deaths, estimates 
of completeness of reporting for both births and deaths are in excess of 99.8 per cent. The population under study, therefore, consists of all deliveries of over 20 weeks' gestation, with the sole selection criterion of notification and/or residence within the jurisdiction of upstate New York (the entire state, excluding New York City).

\section{ZYGosity Estimation}

The twin method is based on differential concordance between the two zygosity classes. Within the present context, sex composition is the sole criterion available on birth records whereby monozygosity can be ruled out. Pairs discordant for sex (male-female) are dizygotic, while concordant pairs may be of either zygosity type. Utilizing this assumption, Weinberg (1902) proposed that the number of dizygotic pairs out of all pairs should be taken as twice the number of sex-discordant pairs. A minor modification in the estimating procedure is effected if it is assumed that sex is a binomial type characteristic, i.e. male or female sex determinations within dizygotic pairs occur independently and with constant probability. Letting $p$ be the probability that a zygote is male, and $\theta$ the proportion of pairs dizygotic, the probabilities of the three sex compositions will be:

\begin{tabular}{|c|c|c|c|c|}
\hline $\begin{array}{c}\text { Sex } \\
\text { Compo- } \\
\text { sition }\end{array}$ & $\begin{array}{c}\text { Relative } \\
\text { Fre- } \\
\text { quency }\end{array}$ & Dizygote & $\begin{array}{l}\text { Mono- } \\
\text { zygote }\end{array}$ & Total \\
\hline $\begin{array}{l}\text { MM } \\
\text { MF, FM } \\
\text { FF } \quad .\end{array}$ & $\begin{array}{l}a \\
b \\
c\end{array}$ & $\begin{array}{c}p^{2} \theta \\
2 p q \theta \\
q^{2} \theta\end{array}$ & $\begin{array}{l}p(1-\theta) \\
q(1-\theta)\end{array}$ & $\begin{array}{c}p(1-q \theta) \\
2 p q \theta \\
q(1-p \theta)\end{array}$ \\
\hline Total .. & 1 & $\theta$ & $(1-\theta)$ & 1 \\
\hline
\end{tabular}

where $p=$ probability that a zygote is male

$q=1-p$

$\theta=$ proportion of pairs dizygotic.

Likelihood estimates of the two parameters are given by:

$$
\begin{aligned}
p & =a+\frac{1}{2} b \\
\theta & =\frac{2 b}{1-(a-c)^{2}} . . .
\end{aligned}
$$

The corresponding Weinberg estimate for the proportion dizygotic is $\theta=2 b$, which coincides with the likelihood estimate when the numbers of MM and FF pairs are the same.

The validity of the estimating procedure depends on assumptions which are not subject to direct empirical confirmation. The sex ratio may differ for monozygotic and dizygotic twins, or the male probability may vary between or within pairs. The extensive literature on the sex ratio in man would imply that a multiplicity of factors influences the probability of maleness. The existence of a positive as well as a negative correlation in the sex of siblings has been suggested. For present purposes, only the ranges of such departures from the binomial are of central importance, and these tend to be of small enough magnitude not to affect the estimates seriously.

A measure of concordance has been presented in a previous publication (Gittelsohn and Milham, 1964). Under the assumption that the probability that a child is affected with a given trait is constant, $p$, and the correlation between sibs is constant, $r$, the number of affected children within a twin pair will be

\begin{tabular}{|c|c|c|}
\hline $\begin{array}{l}\text { Number } \\
\text { Affected }\end{array}$ & $\begin{array}{l}\text { Observed } \\
\text { Frequency }\end{array}$ & Probability \\
\hline $\begin{array}{l}2 \\
1 \\
0\end{array}$ & $\begin{array}{l}a \\
b \\
c\end{array}$ & $\begin{array}{c}p^{2}+p q r \\
2 p q-2 p q r \\
q^{2}+p q r\end{array}$ \\
\hline Total .. & 1 & 1 \\
\hline
\end{tabular}
represented as:

Maximum likelihood estimates of the parameters are:

$$
\begin{aligned}
& p=a+\frac{1}{2} b \\
& r=1-\frac{2 b}{1-(a-c)^{2}} . . . \\
& \text {. . . }
\end{aligned}
$$

The parameter $r$ will be taken as a measure of concordance.

It may be shown that the previously mentioned zygosity estimation procedure is closely related, the proportion monozygotic $(1-\theta)$ having the identical estimator as the concordance coefficient $r$. This is another way of saying that monozygosity is estimated on the basis of sex concordance within pairs. In most twin studies truncate selection is used, and twin pairs with neither member affected do not enter the sample. The present method, based on population data, includes the three classes with nil, one, and two affected within each twin pair. Hence a better measure may be obtained of the incidence of given characteristics and of concordance than is usually available.

\section{Results AND Discussion}

\section{SURVIVORSHIP}

Examination of the frequencies of nil, one, and two foetal deaths among twin pairs reveals that double losses occur more often than would be expected under 
independence of survivorship probabilities for the two members of the pair. This dependence may be interpreted in terms of the existence of heterogeneity of parental pairs, or in terms of "statistical contagion". The latter would imply that the fate of one twin affects the other, while the former effect would arise out of variability in the shared heredity and maternal environment. The fact that the proportion of double stillbirths among like-sexed twins exceeds the proportion among mixed-sexed pairs suggests that more than maternal heterogeneity is at work. The implication is that the foetal mortality of monozygotes is more closely linked than that of dizygotes. This circumstance may be based on the identical genotypes of monozygotes, or on their more intimate foetal relationships.

During the period $1950-60$ there were 21,128 pairs of twins reported with at least 20 weeks' gestation. Table I sets out basic information concerning the twin pairs, specifying for each the sex composition and joint survivorship status of individual members through the first year of life. To achieve brevity, deaths were categorized into four periods:

(1) foetal death (stillborn);

(2) liveborn, dying in less than 1 day;

(3) liveborn, dying in less than 1 month;

(4) liveborn, dying at from 1 to 11 months of age.

There were 911 twin pairs with one stillborn and one liveborn member, and 418 pairs where both twin members were stillborn. In terms of individuals, the percentage stillborn in the entire sample of twins was
4.1. The percentage stillborn among single births during the same period was $1 \cdot 5$.

Among the 19,799 pairs where both twins were liveborn, there were 1,047 pairs with one dying and 1,174 pairs with both dying during the neonatal period (under 28 days). The neonatal death rate for twin individuals was 8.6 per cent., over four times the rate for single births (1.9 per cent.). Of the 911 liveborn members of twin pairs with one live and one stillbirth, 271 (30 per cent.) died during the neonatal period. In general, such pairs were born at an early gestational age. Hence, one of every eight births in twin maternities was a perinatal death. Clearly, pregnancy wastage in plural births constitutes a significant medical and public health problem.

The higher perinatal loss rates among like-sexed pairs than among unlike-sexed pairs points to a selection against the monozygotes (Fig. 1, opposite). The differential loss was greatest during the pre-natal period, where the percentage of children stillborn among the MM and FF pairs was more than double that among the unlike-sexed pairs $(5 \cdot 2$ per cent. and $4 \cdot 8$ per cent. as against $2 \cdot 3$ per cent.). Among pairs with both members liveborn the differential in neonatal death rates was less striking: 10.6 per cent. for individuals for MM pairs, $8 \cdot 1$ per cent. for $F F$, and 8.1 per cent. for MF. Among pairs with both individuals surviving to 1 month, no differential in mortality up to 1 year of age was observed: 0.9 per cent. for MM, 0.8 per cent. for $F F$, and 1.0 per cent. for MF.

TABLE I

TWINS BY OUTCOME, SURVIVORSHIP, AND SEX UPSTATE NEW YORK, 1950-60

\begin{tabular}{|c|c|c|c|c|c|c|c|c|c|c|c|c|}
\hline \multirow{2}{*}{\multicolumn{7}{|c|}{ Survivorship }} & \multirow{3}{*}{$\begin{array}{r}\text { Total } \\
\text { Pairs } \\
21,128 \\
418 \\
911 \\
211 \\
60 \\
5 \\
635\end{array}$} & \multirow{3}{*}{$\begin{array}{r}\text { MM } \\
\text { Pairs } \\
7,300 \\
198 \\
361 \\
95 \\
23 \\
2 \\
241\end{array}$} & \multirow{3}{*}{$\begin{array}{r}\text { FF } \\
\text { Pairs }\end{array}$} & \multicolumn{3}{|c|}{ MF Pairs } \\
\hline & & & & & & & & & & \multirow{2}{*}{$\begin{array}{r}\text { Total } \\
6,818 \\
49 \\
216 \\
41 \\
14 \\
2 \\
159 \\
\end{array}$} & \multirow{2}{*}{$\begin{array}{r}\text { M Dies } \\
116 \\
19 \\
5 \\
1 \\
91\end{array}$} & \multirow[b]{2}{*}{$\begin{array}{r}\text { F Dies } \\
100 \\
22 \\
9 \\
1 \\
68\end{array}$} \\
\hline $\begin{array}{c}\text { Total Number of Twin Pairs } \\
\text { Both twins stillborn } \\
\text { One twin stillborn . } \\
\text { One dies } 1 \text { st day } \\
\text { One dies } 1-27 \text { days } \\
\text { One dies } 1-11 \text { mths } \\
\text { One survives 1st yr }\end{array}$ & $\begin{array}{l}\ldots \\
\cdots \\
\cdots \\
\cdots \\
\cdots\end{array}$ & $\begin{array}{l}\cdots \\
\cdots \\
\cdots \\
\cdots \\
\cdots \\
\cdots\end{array}$ & $\begin{array}{l}\cdots \\
\cdots \\
\cdots \\
\cdots \\
\cdots\end{array}$ & $\begin{array}{l}\cdots \\
\cdots \\
\cdots \\
\cdots \\
\cdots\end{array}$ & $\begin{array}{l}. \\
\cdots \\
\cdots \\
\cdots \\
\cdots\end{array}$ & $\begin{array}{l}\cdots \\
\because \\
\cdots \\
\cdots \\
\cdots\end{array}$ & & & & & & \\
\hline $\begin{array}{c}\text { Both Twins Liveborn } \\
\text { Both die 1st day } \\
\text { One twin dies 1st day } \\
\text { One dies } 1-27 \text { days } \\
\text { One dies } 1-11 \text { mths } \\
\text { One survives 1st yr }\end{array}$ & $\begin{array}{l}. \\
\cdots \\
\cdots \\
\cdots \\
\cdots\end{array}$ & $\begin{array}{l}\cdots \\
\cdots \\
\cdots \\
\cdots\end{array}$ & $\begin{array}{l}\cdots \\
\cdots \\
\cdots \\
\cdots \\
\cdots\end{array}$ & $\begin{array}{l}\ldots \\
\cdots \\
\cdots \\
\cdots\end{array}$ & $\begin{array}{l}\cdots \\
\because \\
\cdots \\
\cdots\end{array}$ & $\begin{array}{l}\cdots \\
\cdots \\
\cdots \\
\cdots \\
\cdots\end{array}$ & $\begin{array}{r}19,799 \\
721 \\
595 \\
232 \\
15 \\
348\end{array}$ & $\begin{array}{r}6,741 \\
334 \\
205 \\
82 \\
7 \\
116\end{array}$ & $\begin{array}{r}6,505 \\
211 \\
210 \\
80 \\
4 \\
126\end{array}$ & $\begin{array}{r}6,553 \\
176 \\
180 \\
70 \\
4 \\
106\end{array}$ & $\begin{array}{r}105 \\
39 \\
2 \\
64\end{array}$ & $\begin{array}{r}75 \\
31 \\
2 \\
42\end{array}$ \\
\hline $\begin{array}{l}\text { Both Twins Survive 1st day } \\
\text { Both die 1-27 days } \\
\text { One twin dies } 1-27 \text { days } \\
\text { One dies } 1-11 \text { mths } \\
\text { One survives } 1 \text { st yr }\end{array}$ & $\begin{array}{l}\cdots \\
\cdots \\
\cdots \\
\cdots\end{array}$ & $\begin{array}{l}\cdots \\
\cdots \\
\cdots \\
\cdots\end{array}$ & $\begin{array}{l}\cdots \\
\cdots \\
\cdots \\
\cdots\end{array}$ & $\begin{array}{l}\cdots \\
\cdots \\
\cdots\end{array}$ & $\begin{array}{l}\cdots \\
\because \\
\cdots \\
\cdots\end{array}$ & $\begin{array}{l}\cdots \\
\cdots \\
\cdots \\
\cdots\end{array}$ & $\begin{array}{r}18,483 \\
221 \\
684 \\
14 \\
670\end{array}$ & $\begin{array}{r}6,202 \\
96 \\
263 \\
5 \\
258\end{array}$ & $\begin{array}{r}6,084 \\
74 \\
197 \\
7 \\
190\end{array}$ & $\begin{array}{r}6,197 \\
51 \\
224 \\
2 \\
222\end{array}$ & $\begin{array}{r}145 \\
1 \\
144\end{array}$ & $\begin{array}{r}79 \\
1 \\
78\end{array}$ \\
\hline $\begin{array}{l}\text { Both Twins Survive 1st mth } \\
\text { Both die 1-11 mths } \\
\text { One twin dies 1-11 mths } \\
\text { Both survive 1st yr .. }\end{array}$ & $\begin{array}{l}\cdots \\
\cdots \\
\cdots\end{array}$ & $\begin{array}{l}\cdots \\
\cdots \\
\cdots\end{array}$ & $\begin{array}{l}\cdots \\
\cdots \\
\cdots\end{array}$ & $\begin{array}{l}\because \\
\because \\
\cdots\end{array}$ & $\begin{array}{l}\because \\
\because \\
\cdots\end{array}$ & $\begin{array}{l}\cdots \\
\cdots \\
\cdots\end{array}$ & $\begin{array}{r}17,578 \\
3 \\
316 \\
17,259\end{array}$ & $\begin{array}{r}5,843 \\
1 \\
106 \\
5,736\end{array}$ & $\begin{array}{r}5,813 \\
0 \\
91 \\
5,722\end{array}$ & $\begin{array}{r}5,922 \\
2 \\
119 \\
5,801\end{array}$ & 69 & 50 \\
\hline
\end{tabular}




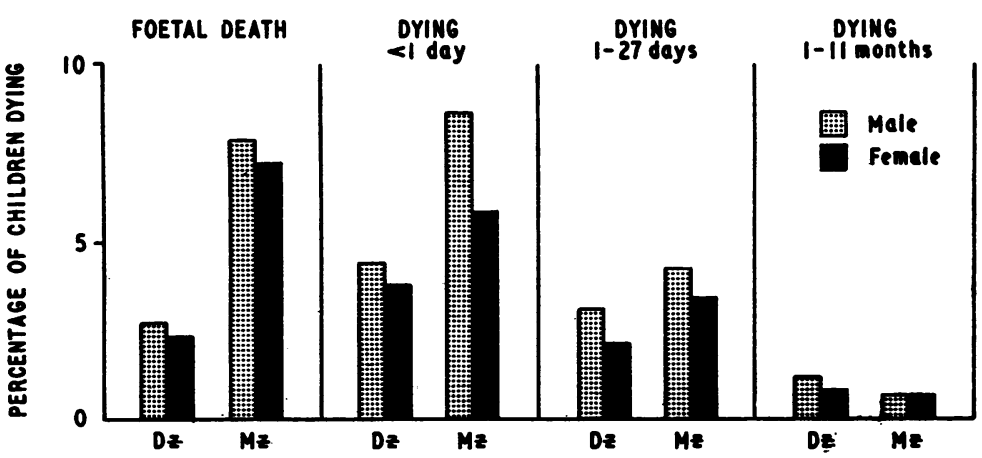

Fig. 1.- Estimated death rates for twins, by sex and zygosity (upstate New York, 1950-60).

Table II illustrates the application of the preceding methods (Equations 1 and 2 above) to the estimation of foetal and neonatal loss rates by sex and zygosity group among the total sample of $N=21,128$ twin pairs recorded in upstate New York during the study period. On the basis of sex composition of the pairs, the proportions male and dizygotic were estimated to be $p=0 \cdot 56069$, and $\theta=0.6458$. The numbers of dizygotic MM and FF pairs were taken to be $N p^{2} \theta$ and $N q^{2} \theta$, respectively, and the numbers of monozygotic MM and FF pairs obtained by subtraction.

Among the 6,818 MF pairs, the observed foetal mortality rate for male members was 2.4 per cent., and for female members $2 \cdot 2$ per cent. The concordance for the binary trait of stillbirth was $r=0 \cdot 296$. Assuming that mortality of individuals in dizygotic twin pairs is not influenced by the sex of the co-twin, these rates were applied to the estimated members of MM and FF dizygotic pairs obtained previously. Hence, the dizygotic MM pairs with two, one, and nil foetal deaths were taken to be proportionate to the three terms of the modified binomial:

\begin{tabular}{c|c}
\hline Number Affected & Expected Proportion \\
\hline 2 & $p_{m}\left(p_{m}+q_{m} r\right)$ \\
1 & $2 p_{m} q_{m}(1-r)$ \\
0 & $q_{m}\left(q_{m}+p_{m} r\right)$ \\
\hline
\end{tabular}

where $p_{m}=$ probability of being affected, given that sex is male.

$q_{m}=$ probability of not being affected, given that sex is male.

$r=$ correlation between twins in pair.

The numbers of monozygotic MM pairs with two, one, and nil losses were obtained by subtraction. The numbers of FF pairs were partitioned in the same fashion, using the parameters $r$ and $p_{f}$ (probability of being affected, given sex is female). Clearly, the procedure depends on two major assumptions which cannot be tested directly and only a "reasonableness" criterion can be applied. By observation, the individuals in the like-sexed pairs experienced foetal loss rates more than double those in unlike-sexed pairs $(5 \cdot 2$ per cent. as against $2 \cdot 4$ per cent. for males; and

TABLE II

OBSERVED AND ESTIMATED FOETAL AND INFANT DEATH RATES BY SEX COMPOSITION AND ZYGOSITY UPSTATE NEW YORK, 1950-60

\begin{tabular}{|c|c|c|c|c|c|c|c|c|c|c|c|}
\hline \multirow{2}{*}{\multicolumn{6}{|c|}{ Joint Survivorship }} & \multirow{3}{*}{$\begin{array}{c}\text { Zygosity } \\
\mathbf{D z} \\
\mathbf{M z}\end{array}$} & \multirow{3}{*}{$\begin{array}{c}\text { Total Pairs } \\
\mathbf{1 3 , 6 4 4} \\
\mathbf{7 , 4 8 4}\end{array}$} & \multicolumn{2}{|c|}{ Number Dying } & \multirow{3}{*}{$\begin{array}{c}\begin{array}{c}\text { Deaths } \\
\text { per } 1,000\end{array} \\
23 \\
75\end{array}$} & \multirow{3}{*}{$\begin{array}{c}\begin{array}{c}\text { Concordance } \\
\text { (r) }\end{array} \\
\begin{array}{c}0 \cdot 30 \\
0 \cdot 54\end{array}\end{array}$} \\
\hline & & & & & & & & \multirow{2}{*}{\begin{tabular}{|l|} 
One \\
433 \\
478
\end{tabular}} & \multirow{2}{*}{$\begin{array}{r}\text { Two } \\
97 \\
321\end{array}$} & & \\
\hline All Pairs .. & $\ldots$ & .. & $\ldots$ & Foetal Death & $\ldots$ & & & & & & \\
\hline Both Liveborn & . & . & .. & Death 1st day & $\ldots$ & $\begin{array}{l}\mathbf{D z} \\
\mathbf{M z}\end{array}$ & $\begin{array}{r}13,108 \\
6,691\end{array}$ & $\begin{array}{l}360 \\
235\end{array}$ & $\begin{array}{l}351 \\
369\end{array}$ & $\begin{array}{l}40 \\
73\end{array}$ & $\begin{array}{l}0.65 \\
0.72\end{array}$ \\
\hline \multicolumn{2}{|c|}{ Both Survive 1st day } & . & $\ldots$ & Death 1st mth & $\ldots$ & $\begin{array}{l}\mathbf{D z} \\
\mathbf{M z}\end{array}$ & $\begin{array}{r}12,395 \\
6,088\end{array}$ & $\begin{array}{l}449 \\
235\end{array}$ & $\begin{array}{l}102 \\
119\end{array}$ & $\begin{array}{l}26 \\
39\end{array}$ & $\begin{array}{l}0 \cdot 29 \\
0.48\end{array}$ \\
\hline \multicolumn{2}{|c|}{ Both Survive 1st mth } & .. & .. & Death 1st yr & $\ldots$ & $\begin{array}{l}\mathbf{D z} \\
\mathbf{M z}\end{array}$ & $\begin{array}{r}11,824 \\
5,734\end{array}$ & $\begin{array}{r}238 \\
78\end{array}$ & $\begin{array}{l}3 \\
1\end{array}$ & $\begin{array}{r}10 \\
7\end{array}$ & $\begin{array}{l}0.02 \\
0.02\end{array}$ \\
\hline
\end{tabular}


$4 \cdot 8$ per cent. as against $2 \cdot 2$ per cent. for females). The excess was primarily found in the class of double losses, a circumstance reflected in the higher concordance coefficient $(r)$ for the total MM and FF pairs. Since like-sexed pairs are of both zygosity types, while unlike-sexed pairs are dizygotic only, it is reasonable that the loss rate differentials are understatements. The assumption of a constant concordance for the three types of dizygotic twinships permits estimation of mortality in dizygotic MM and FF pairs. On such a basis the estimated stillbirth rates were found to be more than three times as high among monozygotic as among dizygotic twins of like sex ( $7 \cdot 8$ per cent. as against 2.4 per cent. for males; and $7 \cdot 2$ per cent. as against $2 \cdot 2$ per cent. for females).

The sex ratio (proportion male) of members of twin pairs, including both live and stillborn, was $0 \cdot 507$. This is somewhat lower than the sex ratio for all livebirths $(0 \cdot 513)$. If attention is restricted to complete pairs where both were liveborn, where both survived to the first day, to the first month, and to the first year of life, a progressive drop is revealed in the male proportion to 0.500 at one year of age. Similarly, the sex concordance (proportion monozygotic) progressively decreases. This would indicate a selection against males and a selection against monozygotic twins during the perinatal period. In the period between one month and one year of life there is no evident selection against monozygotic twins, while the sex differential is greatly decreased.

Table III condenses the information presented in Table I, and shows the higher perinatal loss rates in monozygotic twins compared with dizygotic twins.
Fig. 1 illustrates these findings and shows also that males have consistently higher perinatal mortality rates than females within the same zygosity and survivorship class.

\section{BirTHWEIGHT}

Table IV and Fig. 2 (opposite) present foetal and perinatal mortality rates by birthweight for single and twin individuals. Nearly two-thirds of the twins, as contrasted with 7 per cent. of the single births, weighed less than $2,500 \mathrm{~g}$. at birth. Male twins were heavier than female twins, and twins in MF pairs were heavier than twins in like-sexed pairs. One possibility is that monozygotic twins have shorter gestation times than dizygotic twins, a circumstance which would explain the perinatal mortality differential.

For each weight group under 2,500 g., foetal and perinatal mortality is consistently and substantially lower for twins than for single births of comparable weight (Fig. 3, overleaf). Below 1,000 g., fewer than 4 per cent. of either group survived to one month of age. For the weight class 1.0 to $1.5 \mathrm{~kg}$., one of every four single births died in utero, as contrasted with one in thirteen twin births. Between 1.5 and $2.0 \mathrm{~kg}$., mortality was over four times higher among singles than among twins. Between 2.0 and $2.5 \mathrm{~kg}$., the ratio was over two times. Above $2,500 \mathrm{~g}$., the situation was reversed, with the twins experiencing mortality rates almost double those of the single births. These findings are consistent with previous reports associating mortality, birthweight, and plurality.

Several explanations have been proposed to

TABLE III

ESTIMATED DEATH RATES AND CONCORDANCE BY ZYGOSITY AND JOINT SURVIVORSHIP OF TWINS UPSTATE NEW YORK, 1950-60

\begin{tabular}{|c|c|c|c|c|c|c|c|c|c|}
\hline \multirow{3}{*}{ Survivorship } & \multirow{3}{*}{$\begin{array}{c}\text { Sex } \\
\text { Ratio }\end{array}$} & \multirow{3}{*}{$\underset{\text { Sex }}{\text { Concordance }}$} & \multicolumn{3}{|c|}{ Observed } & \multicolumn{4}{|c|}{ Estimated } \\
\hline & & & \multirow{2}{*}{$\mathbf{M F}$} & \multirow{2}{*}{$\mathbf{M M}$} & \multirow{2}{*}{ FF } & \multicolumn{2}{|c|}{ Dizygotic } & \multicolumn{2}{|c|}{ Monozygotic } \\
\hline & & & & & & $\mathbf{M M}$ & FF & $\mathbf{M M}$ & FF \\
\hline $\begin{array}{ccc}\text { Total Number of } & \text { Twin Pairs } \\
1 \text { stillbirth } & \ldots & \ldots \\
2 \text { stillbirths } & \ldots & \ldots \\
\text { Stillbirth rate } & \ldots\end{array}$ & 0.50686 & 0.354 & $\begin{array}{r}6,818 \\
216 \\
49 \\
0 \cdot 023\end{array}$ & $\begin{array}{r}7,300 \\
361 \\
198 \\
0 \cdot 052\end{array}$ & $\begin{array}{r}7,010 \\
334 \\
171 \\
0 \cdot 048\end{array}$ & $\begin{array}{r}3,507 \\
117 \\
26 \\
0 \cdot 024\end{array}$ & $\begin{array}{r}3,319 \\
100 \\
22 \\
0 \cdot 022\end{array}$ & $\begin{array}{r}3,793 \\
244 \\
172 \\
0 \cdot 078\end{array}$ & $\begin{array}{r}3,691 \\
234 \\
149 \\
0.072\end{array}$ \\
\hline $\begin{array}{c}\text { Both Twins Liveborn } \\
1 \text { death 1st day } \\
2 \text { deaths 1st day } \\
\text { Death rate } \quad .\end{array}$ & 0.50596 & 0.338 & $\begin{array}{r}6,553 \\
180 \\
176 \\
0.041\end{array}$ & $\begin{array}{r}6,741 \\
205 \\
334 \\
0.065\end{array}$ & $\begin{array}{r}6,505 \\
210 \\
211 \\
0.049\end{array}$ & $\begin{array}{r}3,356 \\
97 \\
95 \\
0 \cdot 043\end{array}$ & $\begin{array}{r}3,199 \\
83 \\
81 \\
0.038\end{array}$ & $\begin{array}{r}3,385 \\
108 \\
239 \\
0.087\end{array}$ & $\begin{array}{r}3,306 \\
127 \\
130 \\
0.059\end{array}$ \\
\hline $\begin{array}{cc}\text { Both Twins Survive } 1 \text { st day } \\
1 \text { death } 1 \text { st } \text { mth } & \ldots \\
2 \text { deaths } 1 \text { st } \text { mth } & \ldots \\
\text { Death rate } \quad . & \ldots\end{array}$ & 0.50319 & $0 \cdot 329$ & $\begin{array}{r}6,197 \\
224 \\
51 \\
0 \cdot 026\end{array}$ & $\begin{array}{r}6,202 \\
263 \\
96 \\
0.037\end{array}$ & $\begin{array}{r}6,084 \\
197 \\
74 \\
0 \cdot 028\end{array}$ & $\begin{array}{r}3,138 \\
136 \\
31 \\
0.032\end{array}$ & $\begin{array}{r}3,060 \\
89 \\
20 \\
0 \cdot 021\end{array}$ & $\begin{array}{r}3,064 \\
127 \\
65 \\
0.042\end{array}$ & $\begin{array}{r}3,024 \\
108 \\
54 \\
0.033\end{array}$ \\
\hline 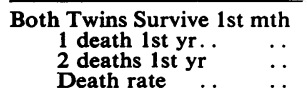 & $0 \cdot 50085$ & $0 \cdot 326$ & $\begin{array}{r}5,922 \\
119 \\
2 \\
0 \cdot 010\end{array}$ & $\begin{array}{r}5,843 \\
106 \\
1 \\
0.009\end{array}$ & $\begin{array}{r}5,813 \\
91 \\
0 \\
0 \cdot 008\end{array}$ & $\begin{array}{r}2,971 \\
69 \\
0 \\
0.012\end{array}$ & $\begin{array}{r}2,951 \\
50 \\
0 \\
0 \cdot 009\end{array}$ & $\begin{array}{r}2,872 \\
37 \\
1 \\
0.007\end{array}$ & $\begin{array}{r}2,862 \\
41 \\
0 \\
0.007\end{array}$ \\
\hline Both Twins Survive 1st yr & 0.50040 & $0 \cdot 327$ & 5,801 & 5,736 & 5,722 & 2,902 & 2,901 & 2,834 & 2,821 \\
\hline
\end{tabular}


TABLE IV

FOETAL, NEONATAL, AND PERINATAL DEATHS AMONG SINGLE AND TWIN INDIVIDUALS BY BIRTHWEIGHT UPSTATE NEW YORK, $1950-60$

\begin{tabular}{|c|c|c|c|c|c|c|c|c|c|c|c|}
\hline \multirow{2}{*}{\multicolumn{6}{|c|}{ Mortality }} & \multirow{3}{*}{$\begin{array}{c}\begin{array}{c}\text { Birth- } \\
\text { weight } \\
\text { (kg.) }\end{array} \\
\text { Total* }\end{array}$} & \multirow{3}{*}{\begin{tabular}{|l}
$\begin{array}{c}\text { Single } \\
\text { Births }\end{array}$ \\
$1,932,111$
\end{tabular}} & \multirow{3}{*}{$\begin{array}{c}\begin{array}{c}\text { Total } \\
\text { Twins }\end{array} \\
42,256\end{array}$} & \multicolumn{3}{|c|}{ Twins born in } \\
\hline & & & & & & & & & \multirow{2}{*}{$\begin{array}{c}\text { MM Pairs } \\
14,600\end{array}$} & \multirow{2}{*}{$\frac{\text { FF Pairs }}{14,020}$} & \multirow{2}{*}{$\frac{\text { MF Pairs }}{13,636}$} \\
\hline Number of births & $\cdots$ & . & $\cdots$ & $\ldots$ & $\cdots$ & & & & & & \\
\hline & & & & & & $\begin{array}{c}<1 \cdot 0 \\
1 \cdot 0 \\
1 \cdot 5- \\
2 \cdot 0- \\
2 \cdot 5+\end{array}$ & $\begin{array}{r}14,398 \\
12,402 \\
23,721 \\
86,215 \\
1,791,029\end{array}$ & $\begin{array}{r}2,363 \\
3,703 \\
10,305 \\
10,864 \\
14,686\end{array}$ & $\begin{array}{r}948 \\
1,321 \\
3,418 \\
3,609 \\
5,139\end{array}$ & $\begin{array}{r}852 \\
1,355 \\
3,577 \\
3,712 \\
4,411\end{array}$ & $\begin{array}{r}563 \\
1,027 \\
3,310 \\
3,543 \\
5,136\end{array}$ \\
\hline \multirow{2}{*}{\multicolumn{3}{|c|}{ Foetal deaths per 1,000 total births }} & \multirow[t]{2}{*}{$\cdots$} & \multirow[t]{2}{*}{$\cdots$} & \multirow[t]{2}{*}{$\cdots$} & Total & $15 \cdot 0$ & $41 \cdot 3$ & $51 \cdot 8$ & $48 \cdot 2$ & $23 \cdot 0$ \\
\hline & & & & & & $\begin{array}{c}<1 \cdot 0 \\
1 \cdot 0 \\
1 \cdot 5- \\
2 \cdot 0- \\
2 \cdot 5+\end{array}$ & $\begin{array}{r}424 \cdot 4 \\
250 \cdot 1 \\
126 \cdot 6 \\
38 \cdot 9 \\
6 \cdot 1\end{array}$ & $\begin{array}{r}283 \cdot 5 \\
77 \cdot 0 \\
24 \cdot 4 \\
17 \cdot 6 \\
11 \cdot 8\end{array}$ & $\begin{array}{r}308 \cdot 0 \\
101 \cdot 4 \\
31 \cdot 3 \\
18 \cdot 0 \\
12 \cdot 6\end{array}$ & $\begin{array}{r}316 \cdot 9 \\
79 \cdot 0 \\
28 \cdot 2 \\
21 \cdot 0 \\
15 \cdot 2\end{array}$ & $\begin{array}{r}191 \cdot 8 \\
42 \cdot 8 \\
13 \cdot 0 \\
13 \cdot 5 \\
8 \cdot 2\end{array}$ \\
\hline \multirow{2}{*}{\multicolumn{4}{|c|}{ Neonatal deaths per 1,000 livebirths }} & \multirow[t]{2}{*}{$\cdots$} & \multirow[t]{2}{*}{$\cdots$} & Total & $16 \cdot 2$ & $90 \cdot 5$ & $110 \cdot 4$ & $86 \cdot 6$ & $73 \cdot 8$ \\
\hline & & & & & & $\begin{array}{c}<1 \cdot 0 \\
1 \cdot 0- \\
1 \cdot 5- \\
2 \cdot 0- \\
2 \cdot 5+\end{array}$ & $\begin{array}{r}944 \cdot 1 \\
547 \cdot 1 \\
199 \cdot 4 \\
42 \cdot 8 \\
5 \cdot 3\end{array}$ & $\begin{array}{r}947 \cdot 4 \\
335 \cdot 0 \\
49 \cdot 1 \\
17 \cdot 0 \\
9 \cdot 0\end{array}$ & $\begin{array}{r}977 \cdot 1 \\
403 \cdot 5 \\
69 \cdot 8 \\
21 \cdot 7 \\
9 \cdot 5\end{array}$ & $\begin{array}{r}936 \cdot 4 \\
282 \cdot 9 \\
37 \cdot 4 \\
12 \cdot 7 \\
9 \cdot 2\end{array}$ & $\begin{array}{r}918 \cdot 7 \\
318 \cdot 4 \\
40 \cdot 7 \\
16 \cdot 9 \\
8 \cdot 2\end{array}$ \\
\hline \multirow{2}{*}{\multicolumn{3}{|c|}{ Perinatal deaths per 1,000 total births. }} & . & & \multirow[t]{2}{*}{$\cdots$} & Total & $30 \cdot 9$ & $128 \cdot 1$ & $156 \cdot 5$ & $130 \cdot 6$ & $95 \cdot 1$ \\
\hline & & & & & & $\begin{array}{c}<1 \cdot 0 \\
1 \cdot 0- \\
1 \cdot 5- \\
2 \cdot 0- \\
2 \cdot 5+\end{array}$ & $\begin{array}{r}967 \cdot 8 \\
660 \cdot 4 \\
300 \cdot 8 \\
80 \cdot 0 \\
11 \cdot 4\end{array}$ & $\begin{array}{r}962 \cdot 3 \\
386 \cdot 2 \\
72 \cdot 3 \\
34 \cdot 3 \\
20 \cdot 7\end{array}$ & $\begin{array}{r}984 \cdot 2 \\
464 \cdot 0 \\
98 \cdot 9 \\
39 \cdot 3 \\
22 \cdot 0\end{array}$ & $\begin{array}{r}956 \cdot 6 \\
339 \cdot 5 \\
64 \cdot 6 \\
33 \cdot 4 \\
24 \cdot 3\end{array}$ & $\begin{array}{r}934 \cdot 3 \\
347 \cdot 6 \\
53 \cdot 2 \\
30 \cdot 2 \\
16 \cdot 4\end{array}$ \\
\hline
\end{tabular}

* Includes births with birthweight not stated.

FOETAL DEATHS

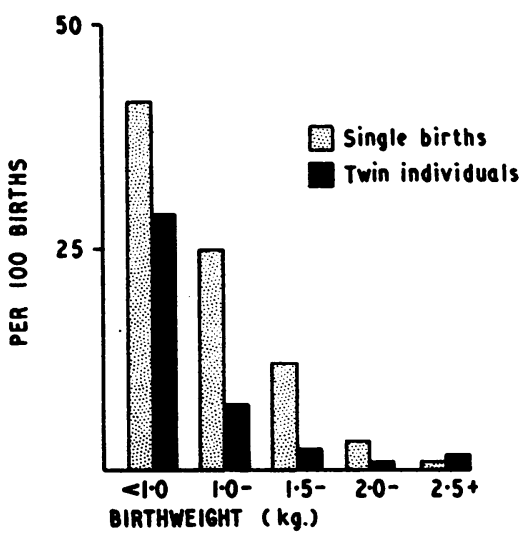

PERINATAL DEATHS

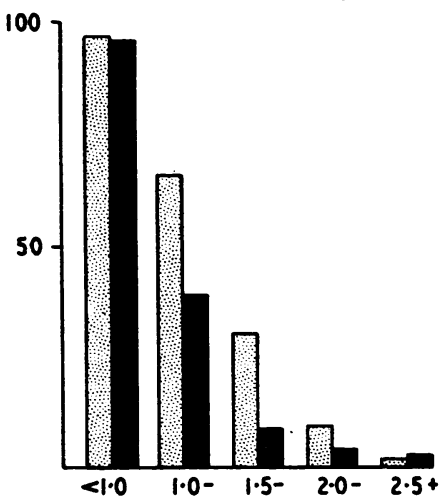

Fig.2.-Foetal and perinatal death rates among single born and twin individuals, by birthweight (upstate New York, 1950-60).

account for the higher mortality of single births than twins at birthweights under $2 \cdot 5 \mathrm{~kg}$. Steiner and Pomerance (1950) attributed the difference to the greater maturity of twins whose gestation length is longer than that of single births of the same weight. Record and others (1952) reported that the inverse association between plurality and mortality persisted when length of gestation was held constant and, therefore, asserted that the maturity argument could not explain the observed differential. Their alternative was that low-weight single births are entirely unrepresentative of the universe of single births, from which they are selected because of complications associated with early delivery and higher mortality.

For birthweights above 2,500 g., mortality increased with plurality-perinatal death rates for twins and single births being $2 \cdot 1$ and $1 \cdot 1$ per cent. respectively, a result consistent with previous reports. 


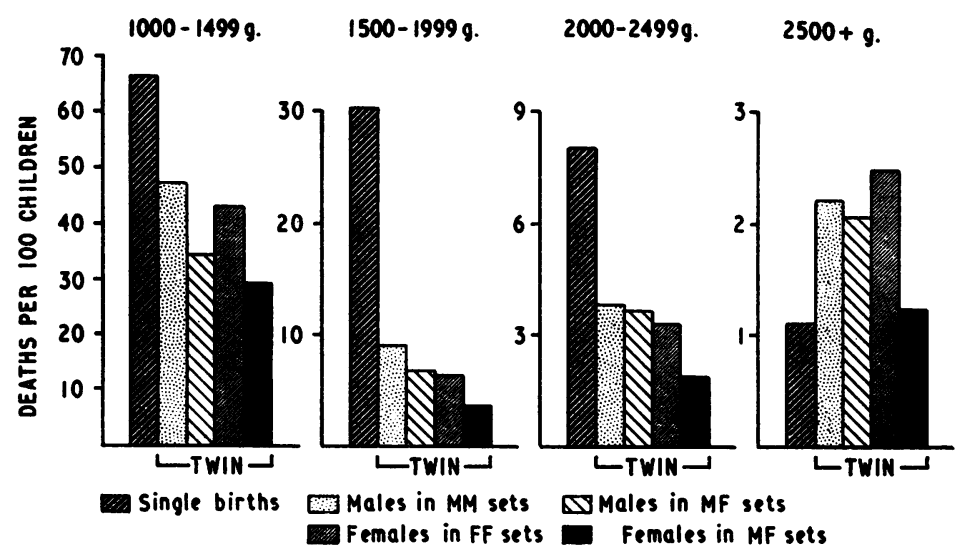

Fig.3.-Perinatal death rates per 100 births by birthweight, sex, and plurality (upstate New York, 1950-60).

One suggestion is that the mortality differential at high birthweight is due to complications in delivering heavy twins.

Perinatal death rates per 1,000 births for twins, by sex composition of pair and birthweight combination, are shown in Table V. Within like-sexed pairs the rate for the lighter twins is exhibited above the rate for the heavier twins, and within MF pairs the rate for males is exhibited above the rate for females. While mortality generally decreased progressively with increasing weight, the weight of the co-twin appeared to be related to survivorship in a complex fashion. For example, the mortality of $2 \mathrm{~kg}$. males in $\mathrm{MM}$ pairs declined by 90 per cent. from 286 to 31, with increasing weight of the co-twin. Similar trends are observed for other classes. The survivorship of fullterm males $(2.5 \mathrm{~kg}$.) in MF pairs depended on the weight of the female co-twin, ranging from 63 when the female was $1.0 \mathrm{~kg}$., to a minimum of 10 when the female was $2.0 \mathrm{~kg}$. The mortality of $1.5 \mathrm{~kg}$. females in FF pairs increases from 46 to 74 to 145 , as the weight of the co-twin increases from 1.5 to over $2 \cdot 5 \mathrm{~kg}$. Evidently, weight disparity between co-twins, along with weight itself, has an important relation to survivorship.

\section{Maternal Age and Birth Rank}

Estimated stillbirth and perinatal loss rates for twins by sex, zygosity, and maternal age are shown in Figs 4 and 5 (opposite). The higher rates for monozygotes are evident at each maternal age.

The zygosity differential is about 3 to 1 for foetal mortality and 2 to 1 for perinatal mortality. No obvious trend with maternal age is in evidence for stillbirth rates in either zygosity class, while survivorship appears to improve with ageing, using the
TABLE V

PERINATAL DEATH RATES (PER 1.000 BIRTHS) AMONG TWINS IN PAIRS CLASSIFIED BY SEX COMPOSITION AND WEIGHT COMBINATIONS UPSTATE NEW YORK, 1950-60

\begin{tabular}{|c|c|c|c|c|c|c|}
\hline Sex & $\begin{array}{l}\text { Weight } \\
\text { (kg.) }\end{array}$ & $<1 \cdot 0$ & $1 \cdot 0$ & $1 \cdot 5-$ & $2 \cdot 0$ & $2 \cdot 5+$ \\
\hline \multirow[t]{5}{*}{ MM Pairs } & $<1 \cdot 0$ & $\frac{992}{992}$ & & & \multicolumn{2}{|c|}{$\frac{\text { Rate for lighter twin }}{\text { Rate for heavier twin }}$} \\
\hline & $1 \cdot 0$ & $\frac{944}{869}$ & $\frac{477}{477}$ & & & \\
\hline & $1 \cdot 5-$ & $\frac{958}{271}$ & $\frac{267}{203}$ & $\frac{85}{85}$ & & \\
\hline & $2 \cdot 0$ & $\frac{1,000}{286}$ & $\frac{583}{100}$ & $\frac{66}{52}$ & $\frac{31}{31}$ & \\
\hline & $2 \cdot 5+$ & $\frac{1,000}{235}$ & $\frac{857}{71}$ & $\frac{176}{70}$ & $\frac{39}{28}$ & $\frac{17}{17}$ \\
\hline \multirow[t]{5}{*}{ FF Pairs } & $<1 \cdot 0$ & $\frac{976}{976}$ & & & \multicolumn{2}{|c|}{$\begin{array}{l}\text { Rate for lighter twin } \\
\text { Rate for heavier twin }\end{array}$} \\
\hline & $1 \cdot 0$ & $\frac{826}{772}$ & $\frac{378}{378}$ & & & \\
\hline & $1 \cdot 5-$ & $\frac{864}{182}$ & $\frac{163}{80}$ & $\frac{46}{46}$ & & \\
\hline & $2 \cdot 0$ & $\frac{856}{428}$ & $\frac{352}{167}$ & $\frac{74}{35}$ & $\frac{25}{25}$ & \\
\hline & $2 \cdot 5+$ & $\frac{1,000}{100}$ & $\frac{421}{210}$ & $\frac{145}{45}$ & $\frac{39}{27}$ & $\frac{21}{21}$ \\
\hline \multicolumn{2}{|l|}{ MF Pairs } & $\begin{array}{l}\text { Weight } \\
<1 \cdot 0\end{array}$ & $\begin{array}{l}\text { of } \mathrm{Ma} \\
1 \cdot 0\end{array}$ & $\begin{array}{l}\text { Twin } \\
1 \cdot 5-\end{array}$ & $2 \cdot 0$ & $2 \cdot 5+$ \\
\hline \multirow{5}{*}{$\begin{array}{l}\text { Weight } \\
\text { of } \\
\text { Female } \\
\text { Twin }\end{array}$} & $<1 \cdot 0$ & $\frac{969}{952}$ & $\frac{800}{740}$ & $\frac{333}{833}$ & $\begin{array}{l}\text { Rate for male twin } \\
\text { Rate for female twin }\end{array}$ & nale twin \\
\hline & $1 \cdot 0$ & $\frac{879}{515}$ & $\frac{417}{315}$ & $\frac{129}{139}$ & $\frac{53}{211}$ & $\frac{63}{688}$ \\
\hline & $1 \cdot 5-$ & - & $\frac{190}{101}$ & $\frac{55}{38}$ & $\frac{34}{26}$ & $\frac{21}{53}$ \\
\hline & $2 \cdot 0$ & - & $\frac{294}{118}$ & $\frac{41}{24}$ & $\frac{37}{19}$ & $\frac{10}{22}$ \\
\hline & $2 \cdot 5+$ & - & - & $\frac{224}{35}$ & $\frac{42}{13}$ & $\frac{23}{11}$ \\
\hline
\end{tabular}




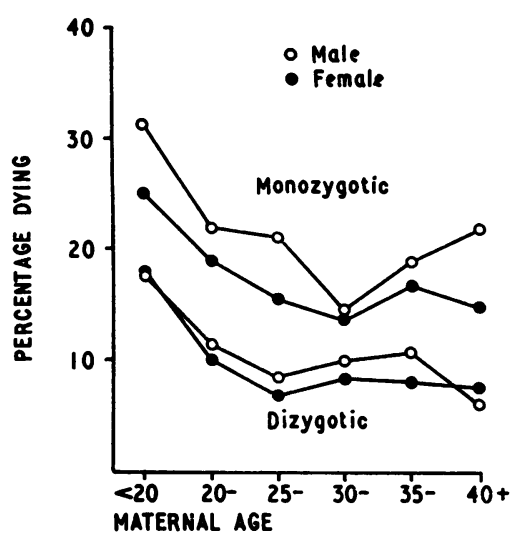

FIG. 4.-Perinatal mortality in twins by sex, zygosity, and maternal age (upstate New York, 1950-60).

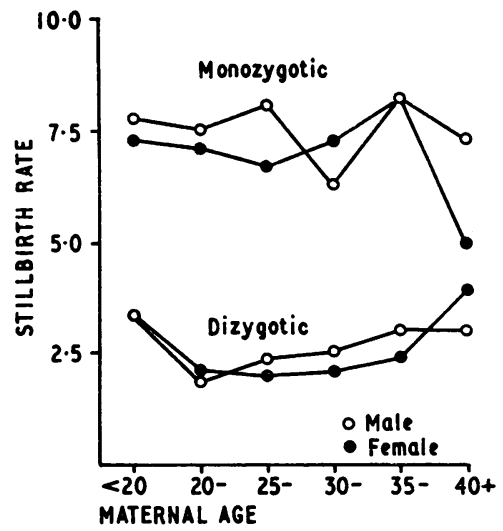

FIG. 5.-Stillbirth rates in twins by sex, zygosity, and maternal age (upstate New York, 1950-60).

criterion of perinatal death. This finding is in contrast with the situation for single births, where perinatal loss rates generally are elevated at the extremes of maternal age.

Fig. 6 presents twins by sex, type of pair, birthweight, and maternal age. The birthweights of twins increase consistently with maternal age. Males in MF pairs are heavier on the average, and females in FF pairs lighter on the average, than males in MM pairs and females in MF pairs at all maternal ages. In part, this may be explained on the basis of the increasing proportion of dizygotic twins in the likesexed sets with increasing maternal age, and on the basis that monozygotic twins are lighter than dizygotic twins.

Obviously, survivorship in twins is more than a simple function of birthweight. Females in MF pairs between 1,500 and 2,500 g. have the lowest perinatal death rates of all twins, while males and females in like-sexed pairs have the highest perinatal loss rates of

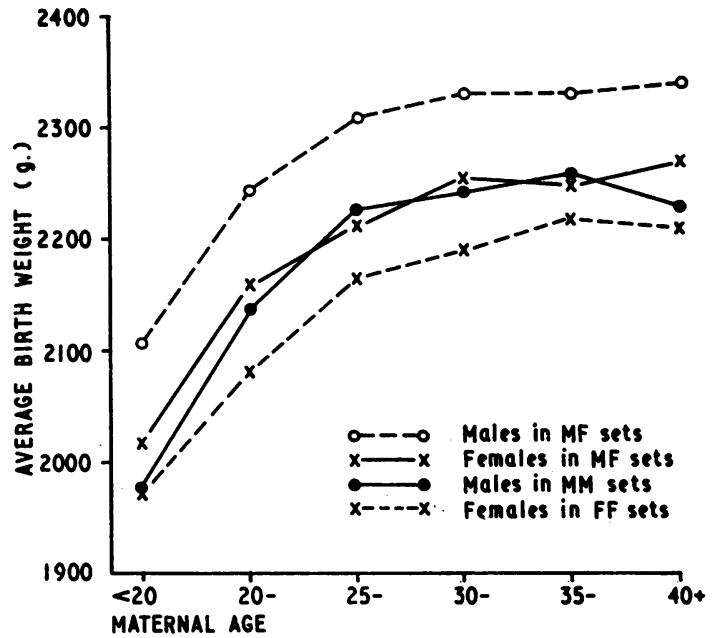

FIG. 6.-Average birthweights of twins by sex, sex composition of twin pair, and maternal age (upstate New York, 1950-60).

all twins. Clearly, sex and zygosity are also important predictors of survivorship and must be considered along with birthweight.

The relationship of the dizygotic twinning rate to maternal age is well demonstrated, and has been the subject of numerous commentaries. Yerushalmy and Sheerar (1940), using American data, and Bulmer (1959), using Italian data, noted a pronounced effect of parity of equal or greater importance than the maternal age effect. In both instances monozygotic rates were found to be unrelated to parity, and only slightly related to maternal age. The present data, based on a larger sample of births, demonstrate a pronounced effect of birth rank on dizygotic twinning rates and a smaller effect on monozygotic rates.

Table VI and Fig. 7 (overleaf) show twinning rates for the two zygosity classes by maternal age and birth order, computed on the basis of the modified Weinberg estimation procedure (Equation 1). For dizygotic twins within each birth order, the trend in the rate by maternal age shows no consistent pattern. The major effect is evidently one of birth rank. For monozygotic twins, the rate increases consistently with increasing birth rank, while for maternal age the pattern is not consistent.

\section{CONCORDANCE}

The twin method has long been used in an attempt to clarify the relative roles of environment and heredity in disease causation. Since monozygotic twins are genetically identical, a higher concordance for a disease or trait in monozygotic, as compared with dizygotic twins, is presumed to mean that the disease or trait has a hereditary component. 
TABLE VI

TWIN MATERNITIES PER 100,000 TOTAL MATERNITIES BY ZYGOSITY GROUP, MATERNAL AGE, AND BIRTH RANK UPSTATE NEW YORK, 1950-60

\begin{tabular}{|c|c|c|c|c|c|c|c|}
\hline \multirow{2}{*}{ Zygosity } & \multirow{2}{*}{$\begin{array}{c}\text { Maternal } \\
\text { Age }\end{array}$} & \multicolumn{6}{|c|}{ Birth Rank } \\
\hline & & Total & $1 \mathrm{st}$ & 2nd & 3rd & 4 th & 5th + \\
\hline & Total & 678 & 308 & 537 & 775 & 1,042 & 1,316 \\
\hline Dizygotic & $\begin{array}{c}<20 \\
20-24 \\
25-29 \\
30-34 \\
35-39 \\
40+\end{array}$ & $\begin{array}{r}313 \\
479 \\
673 \\
887 \\
1,066 \\
583\end{array}$ & $\begin{array}{l}215 \\
280 \\
364 \\
459 \\
522 \\
325\end{array}$ & $\begin{array}{l}595 \\
498 \\
499 \\
640 \\
712 \\
347\end{array}$ & $\begin{array}{r}821 \\
729 \\
758 \\
772 \\
1,022 \\
572\end{array}$ & $\begin{array}{r}927 \\
857 \\
986 \\
1,117 \\
1,204 \\
544\end{array}$ & $\begin{array}{r}* \\
1,567 \\
1,307 \\
1,383 \\
1,355 \\
715\end{array}$ \\
\hline & Total & 365 & 273 & 373 & 399 & 426 & 487 \\
\hline Monozygotic & $\begin{array}{c}<20 \\
20-24 \\
25-29 \\
30-34 \\
35-39 \\
40+\end{array}$ & $\begin{array}{l}326 \\
377 \\
362 \\
380 \\
394 \\
406\end{array}$ & $\begin{array}{l}252 \\
280 \\
263 \\
292 \\
329 \\
379\end{array}$ & $\begin{array}{l}527 \\
407 \\
332 \\
329 \\
368 \\
488\end{array}$ & $\begin{array}{l}645 \\
506 \\
373 \\
364 \\
307 \\
281\end{array}$ & $\begin{array}{l}397 \\
548 \\
444 \\
393 \\
379 \\
317\end{array}$ & $\begin{array}{c}* \\
522 \\
507 \\
485 \\
501 \\
475\end{array}$ \\
\hline
\end{tabular}

* Less than 500 maternities

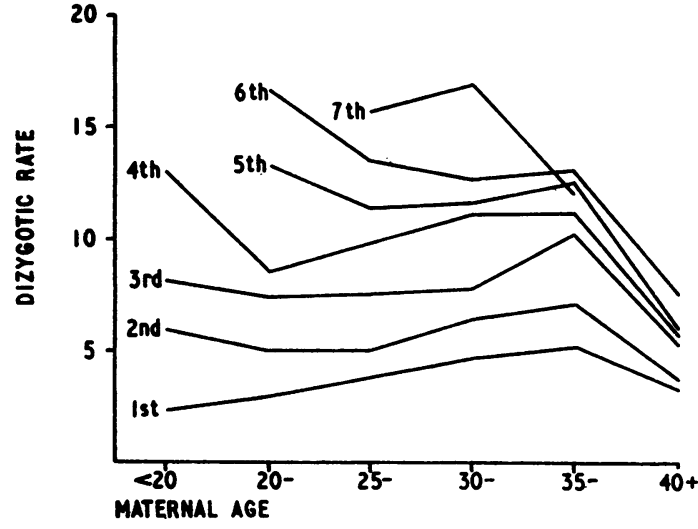

Fig.7.-Dizygotic twin maternities per 1,000 total maternities by birth rank and maternal age (upstate New York, 1950-60).
Using the measure of concordance presented in Equation 2, a number of qualitative characteristics within like-sexed and unlike-sexed twin pairs are analysed in Table VII. In general, concordance is higher in like-sexed pairs as compared with unlikesexed pairs, an expected result, since the former includes the monozygotic pairs. Concordance for foetal death is 0.490 in MM and FF pairs, as con-0 trasted with 0.296 for MF pairs; while perinatal and infant deaths show smaller differences in concordance between the two types of pair. During the pre-natal period the survivorship of members of monozygotic pairs is highly correlated. During the neonatal period the fate of twins within both types of pair is highly correlated, suggesting that non-genetic factors must also be operative.

Congenital anomalies reported on birth records show low concordance for both like and unlike-sexed pairs, the coefficient $r$ being less than $0 \cdot 2$. Although

TABLE VII

TWIN PAIRS BY SEX COMPOSITION AND NUMBER AFFECTED AND CONCORDANCE FOR SELECTED CHARACTERISTICS UPSTATE NEW YORK, $1950-60$

\begin{tabular}{|c|c|c|c|c|c|c|c|c|c|c|}
\hline & Characteristic & & & $\begin{array}{l}\text { Like-Se } \\
\text { imber A }\end{array}$ & $\begin{array}{l}\text { MM, F } \\
\text { d with }\end{array}$ & & & $\begin{array}{l}\text { ike-Se } \\
\text { er Af }\end{array}$ & withir & \\
\hline Death & $\begin{array}{l}\text { Foetal and neonatal } \\
\text { Foetal and } \\
\text { Foetal and infant }\end{array}$ & $\begin{array}{l}\ldots \\
\therefore \\
\therefore\end{array}$ & $\begin{array}{l}13,246 \\
11,656 \\
11,446\end{array}$ & $\begin{array}{r}695 \\
1,192 \\
1,389\end{array}$ & $\begin{array}{r}369 \\
1,462 \\
1,475\end{array}$ & $\begin{array}{l}0.490 \\
0.662 \\
0.623\end{array}$ & $\begin{array}{l}6,553 \\
5,922 \\
5,797\end{array}$ & $\begin{array}{l}216 \\
495 \\
614\end{array}$ & $\begin{array}{r}49 \\
401 \\
407\end{array}$ & $\begin{array}{l}0.296 \\
0.578 \\
0.520\end{array}$ \\
\hline $\begin{array}{c}\text { Congenital } \\
\text { Anomalies }\end{array}$ & 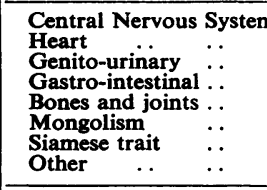 & $\begin{array}{l} \\
\cdots \\
\cdots \\
\cdots \\
\cdots \\
\cdots \\
\end{array}$ & $\begin{array}{l}14,196 \\
14,234 \\
14,278 \\
14,255 \\
14,244 \\
14,304 \\
14,298 \\
14,250 \\
\end{array}$ & $\begin{array}{r}102 \\
70 \\
27 \\
49 \\
59 \\
5 \\
53 \\
\end{array}$ & $\begin{array}{r}12 \\
6 \\
5 \\
6 \\
7 \\
1 \\
12 \\
7\end{array}$ & $\begin{array}{l}0 \cdot 187 \\
0 \cdot 144 \\
0 \cdot 270 \\
0 \cdot 196 \\
0 \cdot 190 \\
0 \cdot 286 \\
1 \cdot 000 \\
0 \cdot 208 \\
\end{array}$ & $\begin{array}{l}\mathbf{6 , 7 7 5} \\
6,791 \\
6,806 \\
6,789 \\
6,771 \\
6,813 \\
6,818 \\
6,793 \\
\end{array}$ & $\begin{array}{r}42 \\
27 \\
12 \\
29 \\
47 \\
5 \\
24 \\
\end{array}$ & $\begin{array}{l}= \\
= \\
= \\
1\end{array}$ & $\begin{array}{l}0.043 \\
0.001 \\
0.000 \\
0.002 \\
0.003 \\
0.000 \\
0.076\end{array}$ \\
\hline$\overline{\text { Erythroblastosi }}$ & . & . & 14,290 & 8 & 12 & 0.750 & 6,812 & 2 & 4 & 0.800 \\
\hline
\end{tabular}


the malformation concordance is low, it is relatively much higher for the like-sexed pairs than for the unlike-sexed pairs. Interestingly, erythroblastosis exhibits the highest concordance in both types of pair. In part, this can be explained on the basis of fathers of erythroblastotic children being more often homozygous for one of the major RH alleles causing histo-incompatibility than other members of the population. However, the number of cases is too small to draw inferences concerning differences in concordance between the two groups of twins. A total of twelve pairs was conjoined at birth (Siamese trait); in all cases both members were of the same sex. This is consistent with the fact that the Siamese trait represents incomplete monozygotic twinning. In this case the concordance coefficient has a value of $1 \cdot 0$.

\section{SUMMARY}

The birth records of 21,128 twin pairs born in the years $1950-60$ in upstate New York are analysed.

The survivorship status of members of monozygotic pairs is more closely linked than that of dizygotic pairs.

Zygosity and sex are important predictors of survivorship in twins. The perinatal mortality of monozygotic twins is greater than that of dizygotic twins, and the mortality of males is greater than that of females, other factors being equal. Males in monozygotic pairs have the poorest survivorship; females in dizygotic pairs have the best survivorship.
The birthweight of twins increases with maternal age.

For birthweights less than $2,500 \mathrm{~g}$., survivorship of twins is better than that of single births. For birthweights greater than $2,500 \mathrm{~g}$., single births have better survivorship than twins.

Birthweight disparity between members of a twin pair, along with weight itself, has an important effect on survivorship.

At constant maternal age the dizygotic and monozygotic twinning rates increases with parity.

Congenital malformations in twins show a low concordance $(r<0 \cdot 2)$, but 43 of 45 concordant pairs were of like sex (MM or FF).

This study was supported by Grant No. CH00080-01 from the United States Public Health Service.

\section{REFERENCES}

Barr, A., and Stevenson, A.C. (1961). Ann. hum. Gen. 25, 131 .

Bulmer, M. G. (1959). Ibid., 23, 454.

Gittelsohn, A. M., and Milham, S. (1964). Amer. J. publ. Hlth, 54, 286.

Karn, M. N. (1953). Acta Genet. med. (Roma), 2, 152.

Lowe, C. R., and Record, R. G. (1951). Brit.J. soc. Med., $5,34$.

Record, R. G., Gibson, J. R., and McKeown, T. (1952). J. Obstet. Gynaec. Brit. Emp., 59, 471.

Steiner, M., and Pomerance, W. (1950). Pediatrics, 6, 872.

Weinberg, W. (1902). Pflügers Arch. ges. Physiol., 88, 346.

Witschi, E. (1952). Cancer Res., 12, 763.

Yerushalmy, J., and Sheerar, S. E. (1940). Hum. Biol., $12,95,247$. 